\title{
Reprodução de Sterna hirundinacea Lesson e S. eurygnatha Saunders (Aves, Laridae), no litoral de Santa Catarina, Brasil
}

\author{
Joaquim Olinto Branco
}

Centro de Ciências Tecnológicas da Terra e do Mar, Universidade do Vale do Itajaí. Caixa Postal 360, 88301-970 Itajaí, Santa Catarina, Brasil. E-mail: branco@cttmar.univali.br

\begin{abstract}
Reproduction of Sterna hirundinacea Lesson and S. eurygnatha Saunders, along Santa Catarina coast, Brazil. On the Brazilian coast, those species nest sympatrically, from the islands of Espírito Santo State to Santa Catarina State. From 1999 to 2002 a sampling program was implanted with the objectives of studying the breeding of S. hirundinacea and S. eurygnatha along Santa Catarina coast. The size of colonies in number of couples, reproduction strategy, length, width, weight and the volume of the eggs, as well as bill length and weight of the nestlings were determined. S. hirundinacea youth weight/length relationship was estimated and the reproductive success of the terns colonies was also determined.
\end{abstract}

KEY WORDS. Conservation, seabirds, terns.

O trinta-réis-de-bico-vermelho Sterna hirundinacea Lesson, 1831 e o trinta-réis-de-bico-amarelo $S$. eurygnatha Saunders, 1876, são duas espécies de ampla distribuição. S. hirundinacea ocorre da Terra do Fogo à Bahia (Brasil) e $S$. eurygnatha da Patagônia até as Antilhas (Sick 1997).

No litoral brasileiro, essas espécies nidificam em simpatria, durante o período de abril a agosto, nas ilhas costeiras dos Estados de Espírito Santo a Santa Catarina (ANTAS 1991, Sick 1997, Efe et al. 2000). A escolha do local de reprodução, pode mudar de um ano para outro, sendo freqüente o abandono em massa das áreas de nidificação (SCOlaro et al. 1996, Yorio et al. 1994, EFE et al. 2000).

A reprodução em colônias mistas, pode atuar como uma adaptação antipredatória para $S$. hirundinacea e $S$. eurygnatha que reproduzem no solo. Apesar de serem freqüentemente citadas nos levantamentos de ornitólogos marinhos brasileiros, existem poucas informações sobre a reprodução dessas trintaréis no litoral do Brasil.

Este trabalho tem por objetivo contribuir para o conhecimento da biologia reprodutiva de $S$. hirundinacea e $S$. eurygnatha, no litoral de Santa Catarina.

\section{MATERIAL E MÉTODOS}

O estudo foi realizado entre os meses de abril a outubro de 1999 a 2002 nas Ilhas Itacolomis, Penha $\left(26^{\circ} 42^{\prime} 36^{\prime \prime}\right.$ $\left.48^{\circ} 37^{\prime} 06^{\prime \prime} \mathrm{W}\right)$, em 1999 e 2002 na Ilha Deserta (Reserva Bioló-

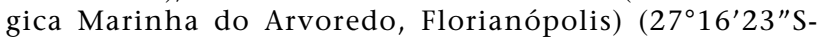
$\left.48^{\circ} 19^{\prime} 53^{\prime \prime} \mathrm{W}\right)$, em 2000 nas Ilhas Moleques do Sul, Florianópolis $\left(27^{\circ} 51^{\prime} \mathrm{S}-48^{\circ} 26^{\prime \prime} \mathrm{W}\right)$ e 2002 na Ilha dos Cardos, Baía Sul, Florianópolis $\left(27^{\circ} 48^{\prime} 55^{\prime \prime} \mathrm{S}-48^{\circ} 34^{\prime} 52^{\prime \prime} \mathrm{W}\right)$.

Em cada sítio de nidificação foi utilizado a mesma rotina de trabalho na qual foi: determinado o tamanho da colônia em número de casais, estratégia de reprodução de Sterna hirundinacea e $S$. eurygnatha, registrado o comprimento total (Lt) e largura (Wid) em centímetros e peso total (Wt) em gramas dos ovos por ninho, bem como o comprimento do cúlmem do bico $\left(\mathrm{Lt}_{\text {bico }}\right.$ ) e peso dos filhotes, capturados manualmente e de alguns adultos encontrados presos em linhas de pesca nas bordas da colônia. Para as medidas de comprimento e largura foram utilizados paquímetro com $0,05 \mathrm{~mm}$ e nos pesos, balança Pesola de 100, 350 e 500 g, com precisão de 1 , 3 e $5 \mathrm{~g}$ respectivamente. Durante os censos, foram registrados a predação dos ovos nas colônias através da observação direta dos predadores.

O volume dos ovos foi estimado de acordo com HoyT (1979) pela equação: $\operatorname{Vol}\left(\mathrm{cm}^{3}\right)=\mathrm{Kv} \cdot \mathrm{Lt} . \mathrm{Wid}^{2}, \operatorname{logo} \mathrm{Kv}=\mathrm{V} / \mathrm{Lt} . \mathrm{Wid}^{2}$, onde $\mathrm{Kv}=$ coeficiente volumétrico; $\mathrm{Lt}=$ comprimento total; Wid = largura do maior eixo do ovo.

O sucesso reprodutivo foi estimado da seguinte maneira: número de juvenis avistados na colônia/número de ninhos com ovos registrados (x 100).

Independente da espécie, as diferentes fases de desenvolvimento dos filhotes foram estabelecidas em função do comprimento do cúlmem do bico, da idade dos jovens anilhados e características da plumagem. Assim, o Jovem I corresponder os filhotes com comprimento de bico entre 1,0 a $1,4 \mathrm{~cm}$ e idade entre 1 a 5 dias, encontrados geralmente nos ninhos. Jovem II com $\mathrm{Lt}_{\text {bico }}$ entre 1,5 a 2,0 cm, entre 6 a 15 dias; nessa fase os filhotes são encontrados perambulando pela colônia. Jovem III com $\mathrm{Lt}_{\text {bico }}$ entre 2,1 a $2,5 \mathrm{~cm}$ e com aproximadamente 16 a 30 dias; filhotes muito ativos e os de $S$. eurygnatha agrupavamse em "creches". Juvenil com $\mathrm{Lt}_{\text {bico }}$ a partir de $2,5 \mathrm{~cm}$, acima de 30 dias de idade, apresentando capacidade de voar a pequena ou média distância. 
O teste ANOVA (SoKal \& Rohlf 1969) foi utilizado para verificar a existência de diferenças significativas entre o comprimento, largura, peso e volume dos ovos entre os sítios de nidificação, bem como, entre o volume dos ovos nos meses de junho, julho e agosto nas Ilhas Moleques do Sul, e entre os anos de estudo nas Ilhas Itacolomis. O contraste das médias através do teste de Tukey-Kramer, quando da ocorrência de diferenças significativas, foi aplicado para indicar a fonte causadora das variações. O teste " $t$ " de Student $(\mathrm{p}<0,05)$ para verificar a existência de diferenças significativas entre as Ilhas Itacolomis e Ilha dos Cardos, no peso dos Jovens I, II e III, Juvenis de $S$. hirundinacea, bem como para o peso dos de $S$. eurygnatha entre as Ilhas Moleques do Sul e Cardos.

\section{RESULTADOS}

No litoral catarinense, $S$. hirundinacea nidificou em simpatria com S. eurygnatha na Ilha Deserta em 1999, nas Ilhas Moleques do Sul em 2000, na Ilha dos Cardos em 2002 e foi a única espécie de trinta-réis a ocupar as Ilhas Itacolomis entre 1999 a 2002 (Tab. I).

O número de pares reprodutores de $S$. hirundinacea variou de acordo com o sítio, investindo entre um a três ovos, com uma média de 1,22 $\pm 0,51$ a 1,44 $\pm 0,54$ ovos por ninho (Tab. I). O comprimento dos ovos não diferiu entre as ilhas $\left(\mathrm{F}_{3-446}=0,7295 ; \mathrm{p}=0,05\right)$, variando em média entre $4,48 \pm 0,23$ a $4,60 \pm 0,18 \mathrm{~cm}$; enquanto que a largura foi significativamente diferente entre as áreas de nidificação $\left(\mathrm{F}_{3-446}=11,4170\right.$; $\mathrm{p}=0,0001)$, sendo que as maiores médias ocorreram na Ilha Deserta $(3,35 \pm 0,23 \mathrm{~cm})$ e as menores na Ilha dos Cardos $(3,24$ $\pm 0,10 \mathrm{~cm}$ ); o peso dos ovos apresentou diferença entre os sítios $\left(\mathrm{F}_{3-427}=6,1540 ; \mathrm{p}=0,0004\right)$, com as maiores médias $(26,95 \pm$ $1,60 \mathrm{~g})$ registradas na tentativa de reprodução de 2002 na Ilha Deserta e as menores $(25,04 \pm 1,85 \mathrm{~g})$ nas Ilhas Moleques do Sul (Tab. I). O sucesso reprodutivo de $S$. hirundinacea nas menores colônias foi estimado entre 65,4 a 66,1 e nas maiores entre 1,3 a $34,1 \%$ (Tab. I).

Sterna eurygnatha esteve representada por um pequeno contingente de reprodutores, investindo em apenas um ovo por ninho, com comprimento significativamente diferente entre as ilhas $\left(\mathrm{F}_{2-97}=8,1620 ; \mathrm{p}=0,0005\right)$, onde as maiores $(5,13$ $\pm 0,27 \mathrm{~cm})$ e as menores $(4,90 \pm 0,31 \mathrm{~cm})$ médias ocorreram, respectivamente na Ilha Deserta e Ilhas Moleques do Sul; a lar- gura dos ovos seguiu a mesma tendência do comprimento, apresentando diferença significativa entre os sítios $\left(\mathrm{F}_{2-97}=\right.$ 15,7360; $\mathrm{p}=0,0001$ ), com valores médios entre 3,45 $\pm 0,26 \mathrm{a}$ $3,83 \pm 0,40 \mathrm{~cm}$ (Tab. I). O peso dos ovos entre as Ilhas Moleques do Sul e Cardos foram diferentes $(t=9,8730 ; p=0,0001$, $\mathrm{gl}=68)$, variando em média entre $26,29 \pm 3,05$ a $34,53 \pm 4,24$ $\mathrm{g}$; apresentando um sucesso reprodutivo estimado entre 24,5 a $72,3 \%$ (Tab. I). Na Ilha Deserta a espécie teve seus ovos predados por Larus dominicanus Lichtenstein, 1823, abandonando em seguida a área de nidificação.

Não foi encontrada diferença significativa entre o volume médio dos ovos de $S$. hirundinacea, quando foram comparados os meses de postura em uma mesma temporada reprodutiva, nas Ilhas Moleques do Sul $\left(\mathrm{F}_{2-397}=1,8910 ; \mathrm{p}=0,05\right)$ (Fig. 1); bem como, entre os anos de reprodução (1999 a 2002) nas Ilhas Itacolomis $\left(\mathrm{F}_{3-253}=0,1916 ; \mathrm{p}=0,05\right)$, os quais apresentaram volume médio variando entre $26,16 \pm 4,14$ a 26,57 \pm $2,04 \mathrm{~cm}^{3}$ (Fig. 2). Entretanto, foi encontrada diferença significativa entre os sítios de nidificação $\left(\mathrm{F}_{3-785}=5,0560 ; \mathrm{p}=0,0019\right)$ (Fig. 3). O contraste das médias, demonstrou que essas diferenças ocorreram, principalmente, na Ilha Deserta, onde foram registradas as maiores médias $(27,35 \pm 5,23)$, enquanto que os menores volumes médios dos ovos foram observados nas Ilhas Moleques do Sul $(25,92 \pm 2,11)$ e Ilha dos Cardos $(25,95 \pm 2,01)$.

O período reprodutivo de $S$. hirundinacea no litoral catarinense pode ser caracterizado em função do registrado na Ilha dos Cardos. Os primeiros adultos foram observados em meados de abril, após o início da postura dos ovos em maio, ocorreu um incremento gradual na população até julho, onde foi registrada a ocorrência de aproximadamente 450 casais, culminando com o pico de desova (Fig. 4). Entre 20 a 25 de maio, nasceram os primeiros filhotes, sendo que a partir de julho ocorreu um acréscimo gradual de jovens na colônia, seguido da redução dos ninhos com ovos. Entre os meses de junho a setembro foi comum a presença de jovens das quatro classes etárias, sendo progressivo o abandono da área de nidificação pelo adultos e juvenis até o início de outubro (Fig. 4).

Observando a estratégia de nidificação de $S$. eurygnatha na Ilha dos Cardos, fica evidente, que essa espécie abre espaço no centro da colônia de $S$. hirundinacea através da agressividade e do maior porte; ocupando a colônia em meados de maio, quando os primeiros ovos da espécie simpátrica já foram colocados.

Tabela I. Esforço reprodutivo de Sterna hirundinacea e S. eurygnatha, nas Ilhas Itacolomis, Deserta, Moleques do Sul e Cardos, Santa Catarina.

\begin{tabular}{|c|c|c|c|c|c|c|c|}
\hline \multirow[b]{2}{*}{ Número médio } & \multirow[b]{2}{*}{ Itacolomis } & \multicolumn{3}{|c|}{ Sterna hirundinacea } & \multicolumn{3}{|c|}{ Sterna eurygnatha ** } \\
\hline & & Deserta & Moleques & Cardos & Deserta & Moleques & Cardos \\
\hline Casais & 280 & 500 & 1200 & 268 & 65 & 200 & 76 \\
\hline Ovos/postura & $1,36 \pm 0,51$ & $1,22 \pm 0,51$ & $1,44 \pm 0,54$ & $1,31 \pm 0,13$ & 1,00 & 1,00 & 1,00 \\
\hline Comprimento dos ovos $(\mathrm{cm})$ & $4,60 \pm 0,18$ & $4,48 \pm 0,23$ & $4,50 \pm 0,21$ & $4,58 \pm 0,19$ & $5,13 \pm 0,27$ & $4,90 \pm 0,31$ & $5,12 \pm 0,16$ \\
\hline Largura dos ovos $(\mathrm{cm})$ & $3,31 \pm 0,12$ & $3,35 \pm 0,23$ & $3,28 \pm 0,10$ & $3,24 \pm 0,10$ & $3,83 \pm 0,40$ & $3,45 \pm 0,26$ & $3,62 \pm 0,13$ \\
\hline Peso dos ovos (g) & $25,93 \pm 2,27$ & * $26,95 \pm 1,60$ & $25,04 \pm 1,85$ & $25,55 \pm 4,20$ & - & $26,29 \pm 3,05$ & $34,53 \pm 4,24$ \\
\hline Sucesso reprodutivo (\%) & 65,4 & 1,3 & 34,1 & 66,1 & 0,0 & 24,5 & 72,3 \\
\hline
\end{tabular}

* Dados da tentativa de reprodução de 2002. Em 1999 não foi registrado o peso dos ovos. ${ }^{* *}$ Não houve registro de S. eurygnatha para a Ilha de Itacolomis.

Revista Brasileira de Zoologia 20 (4): 655-659, dezembro 2003 

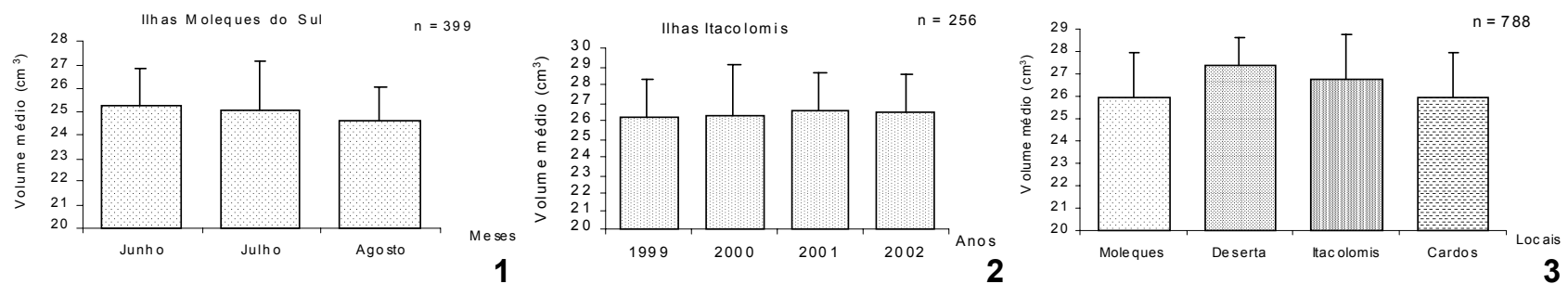

Figuras 1-3. Volume médio dos ovos de Sterna hirundinacea: (1) entre junho a agosto de 2000, nas Ilhas Moleques do Sul; (2) entre os anos de 1999 a 2002, nas Ilhas Itacolomis; (3) nos sítios de reprodução do litoral de Santa Catarina.

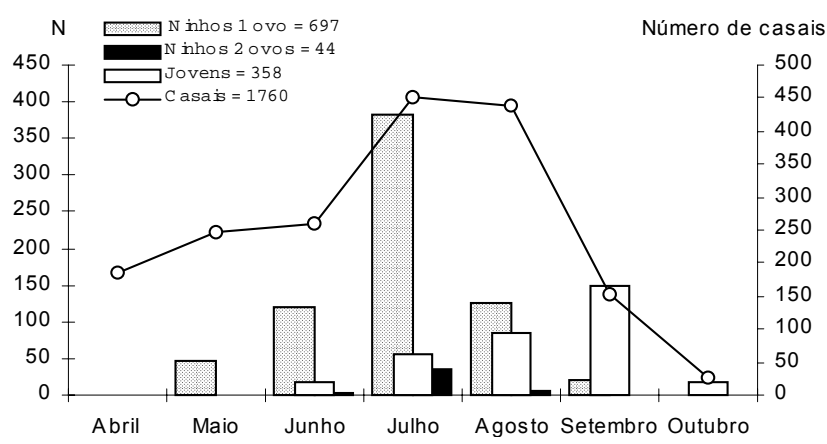

Figura 4. Número de ninhos com um e dois ovos, jovens e casais de Sterna hirundinacea entre abril a outubro de 2002, na llha dos Cardos.

Entre 20 a 25 de junho foram encontrados os primeiros filhotes, após duas a três semanas, esses jovens foram observados em aglomerados (creches) nas bordas da colônia. A partir de agosto ocorreu o deslocamento de adultos e juvenis do sítio de nidificação, sendo que após a segunda semana de setembro não foram registradas a ocorrência de exemplares na Ilha dos Cardos.

O peso corporal de $S$. hirundinacea oscilou em função da idade dos exemplares. Os Jovem I das Ilhas Itacolomis e Ilha dos Cardos não apresentaram diferença significativa entre os pesos médios, ficando em torno de $31,0 \mathrm{~g}$, enquanto que os Jovem II da Ilha dos Cardos foram significativamente mais pesados $(\mathrm{t}=3,617 ; \mathrm{p}=0,0007, \mathrm{gl}=46)$, mas a partir dos Jovem III $(\mathrm{t}=2,704 ; \mathrm{p}=0,0082, \mathrm{gl}=90)$ e dos Juvenil $(\mathrm{t}=3,451 ; \mathrm{p}=$ $0,0015, \mathrm{gl}=34)$, as diferenças significativas foram atribuídas aos maiores pesos dos exemplares das Ilhas Itacolomis (Fig. 5). Embora com peso médio, aparentemente diferente entre adultos das duas ilhas, o número reduzido $(\mathrm{n}=7)$ de trinta-réis e a captura acidental em redes de nylon abandonadas nas ilhas, inviabilizou uma análise mais acurada.

O comprimento do cúlmen do bico e peso dos jovens de S. eurygnatha da Ilha dos Cardos variaram entre $1,23 \pm 0,21 \mathrm{~cm}$ e peso $41,67 \pm 19,35 \mathrm{~g}$ nos Jovem $\mathrm{I}$ a $2,86 \pm 0,13 \mathrm{~cm}$ a $156,14 \pm$ 11,51 g nos Juvenil (Tab. II).

A relação entre o peso corporal e o comprimento do cúlmen do bico de $S$. hirundinacea das Ilhas Itacolomis e Cardos, em conjunto, indica que a espécie apresenta crescimento do tipo alométrico negativo ( $w t=27,5450 \mathrm{Lt}^{1,8490}, \mathrm{r}^{2}=0,8583$ ), com os maiores incrementos em peso ocorrendo nas classes iniciais de comprimento (Fig. 6).

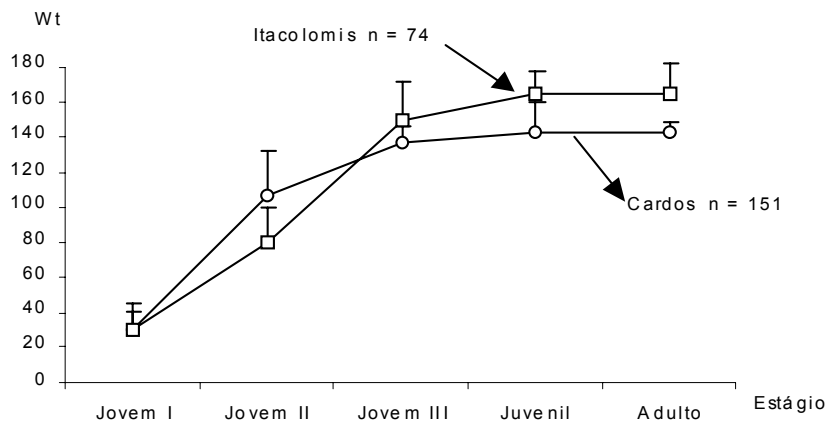

Figura 5. Relação entre o peso e os estágio de Sterna hirundinacea, nas Ilhas Itacolomis e Ilha dos Cardos.

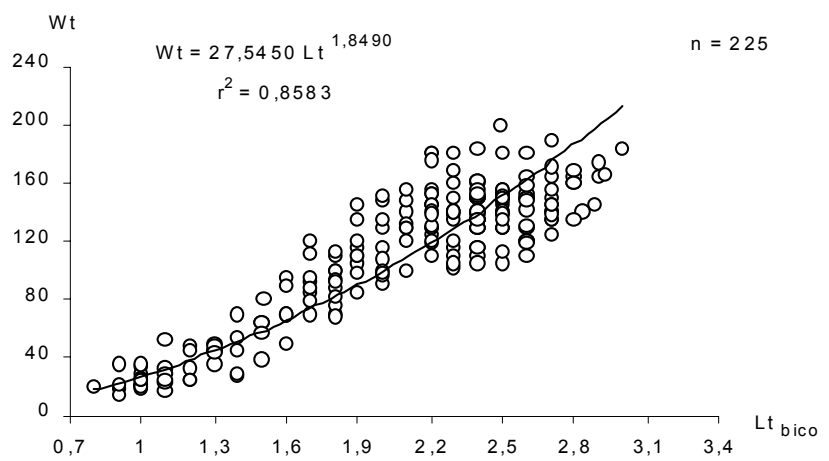

Figura 6. Relação peso comprimento do bico de Sterna hirundinacea, nas llhas Itacolomis e Ilha dos Cardos (dados fundidos).

\section{DISCUSSÃO}

As ilhas costeiras de Santa Catarina são importantes locais de reprodução e refúgio de aves marinhas (BEgE \& PAULI 1988). Entretanto, a escolha do local de nidificação de $S$. hirundinacea e S. eurygnatha pode mudar de um ano para outro; essas aves são consideradas sensíveis a qualquer tipo de distúrbio, sendo freqüente o abandono da área de nidificação (FurNess \& Monaghan 1987, Buckley \& Buckley 1972, Yorio et al. 1994, SCOLARo et al. 1996).

A predação pode resultar na perda do conteúdo dos ni- 
Tabela II. Comprimento do cúlmen do bico e peso dos filhotes de Sterna eurygnatha, nas classes etárias encontrados na Ilha dos Cardos.

\begin{tabular}{|c|c|c|c|c|c|c|c|}
\hline \multirow{2}{*}{ Estágio } & \multirow{2}{*}{$\mathrm{n}$} & \multicolumn{3}{|c|}{ Comprimento do cúlmen $(\mathrm{cm})$} & \multicolumn{3}{|c|}{ Peso (g) } \\
\hline & & $\mathrm{Lt}$ & $<$ & $>$ & Wt & $<$ & $>$ \\
\hline Jovem I & 3 & $1,23 \pm 0,21$ & 1,0 & 1,4 & $41,67 \pm 19,35$ & 30,0 & 64,0 \\
\hline Jovem II & 11 & $1,66 \pm 0,15$ & 1,5 & 1,9 & $74,09 \pm 7,65$ & 61,0 & 85,0 \\
\hline Jovem III & 9 & $2,17 \pm 0,25$ & 2,0 & 2,5 & $126,44 \pm 28,06$ & 95,0 & 180,0 \\
\hline Juvenil & 7 & $2,86 \pm 0,13$ & 2,6 & 3,1 & $156,14 \pm 11,51$ & 145,0 & 178,0 \\
\hline
\end{tabular}

nhos e abandono da área de reprodução por alguns casais ou de toda a colônia, sendo que ação das gaivotas é considerada uma das causas da perda de ovos dos trinta-réis (BucKLey \& BUCKLEY 1972).

A coleta de ovos das aves marinhas era um costume comum entre pescadores e moradores do litoral de Santa Catarina (Bege \& Pauli 1988, Sick 1997). Esse hábito foi praticado nas décadas de 50 a 80; contudo, hoje são raros os relatos de perturbações humanas nas colônias. A alternância entre sítios de nidificação por $S$. hirundinacea e $S$. eurygnatha, atualmente pode ser atribuído a predação da gaivota Larus dominicanus, que causou o abandono da colônia mista da Ilha Deserta em agosto/ 99 , com a sobrevivência de alguns jovens. Nos anos de 2000 e 2002, as duas espécies retornaram à ilha com um contingente menor de reprodutores; após a postura dos ovos em maio e junho ocorreu o abandono da área de nidificação devido a predação total dos ninhos pelas gaivotas. Enquanto que nas Ilhas Itacolomis, S. hirundinacea nidificou regularmente de 1996 a 2002, entre os meses de abril a setembro, com uma população estável de 250 a 280 casais e um sucesso reprodutivo em torno de 63,0 a $68,0 \%$.

Os resultados de Yorio \& Quintana (1997) corroboram as observações acima, onde indicam que $L$. dominicanus foi o principal predador de ovos dos S. maxima Boddaert, 1783 e $S$. eurygnatha em Punta León (Argentina), causando importante perdas ao longo das estações reprodutivas, com as maiores taxas de predação incidindo sobre a menor dessas espécies ( $S$. eurygnatha).

O período reprodutivo de $S$. hirundinacea e $S$. eurygnatha na costa catarinense, está de acordo com o descrito por Sick \& Leão (1965) para a Ilha dos Papagaios (Rio de Janeiro), Baía de Guanabara (SICK 1997), ilhas do litoral Sul do Espírito Santo (EғE et al. 2000) e Ilha Deserta (Santa Catarina) (R. Escalante, T.R. Azevedo e A. Freyesleben, dados não publicados). A nidificação em simpatria dessas espécies, parece possível devido às estratégias similares de reprodução utilizadas. Dessa forma, a associação entre trinta-réis, parece um modo eficiente de reduzir a taxa de predação nas colônias de nidificação (QuiNTANA $\&$ YORIO 1997).

O número médio de ovos por ninho e o sucesso reprodutivo de $S$. hirundinacea nas colônias do litoral catarinense, foram relativamente inferior ao registrado para Punta Loma, Argentina (Scolaro et al. 1996), respectivamente com 1,65 \pm 0,64 e 73,4\%, com um período de incubação entre 21 a 23 dias. Assim como, em S. eurygnatha, que na colônia de Punta León, Argentina, a taxa de predação esteve em torno de $45,3 \%$ (Quintana \& Yorio 1997). Essas diferenças, provavelmente po- dem estar associadas à postura dos ovos durante os meses de inverno (Santa Catarina) e de primavera (Argentina), além da disponibilidade e abundância de alimento, tamanho das colônias e pressão dos predadores, entre outros fatores.

A reprodução em colônia pode atuar como uma vantagem seletiva contra predação em aves marinhas. Em grandes colônias a desova sincronizada dificulta a ação de predadores em usar uma fonte abundante de alimento; além disso, essas colônias atuam como um agrupamento egoísta, onde os ninhos periféricos apresentam as maiores taxas de predação (Coulson 1968, Brown \& Brown 1987, Brunton 1997). Entretanto, o sucesso reprodutivo de $S$. hirundinacea e $S$. eurygnatha no litoral catarinense, foram acentuadamente menor nas maiores colônias, onde nidificaram entre 500 a 1200 casais.

De acordo com BRUNTON (1999), o tamanho considerado "ótimo" para colônias de trinta-réis na América do Norte, suportam a idéia de um tamanho médio, em torno de 150 ninhos; sendo que a proporção de ninhos predados declina com o tamanho da colônia, mas a sobrevivência dos jovens não segue essa tendência. Em geral, a performance reprodutiva das aves marinhas em colônias que suportam grandes populações, demonstram que ocorrem elevada mortalidade de filhotes (HunT et al. 1986).

A predação é um dos principais fatores que afetam o sucesso reprodutivo das aves marinhas e uma força determinante na estratégia utilizada pelas colônias que nidificam no solo (BURGER \& GOCHFELD 1994).

O comprimento do cúlmen do bico e o peso corporal dos trinta-réis, variaram em função da idade e sítios de nidificação dos exemplares; sendo que $S$. hirundinacea apresenta crescimento alométrico negativo. Em geral, a relação peso/ comprimento tem sido utilizada para estimar o peso de um exemplar através do conhecimento do seu comprimento, sendo amplamente empregada em estudos de dinâmica de populações. Essa relação, mostrou-se adequada para indicar a forma de crescimento de $S$. hirundinacea, sendo que a equação resultante, pode ser utilizada para estimar um desses parâmetros, na ausência de paquímetro ou pesola.

Em áreas onde ocorrem grandes concentrações de predadores como gaivotas, urubus e de um a dois casais de gaviões (Ilha Deserta e Ilhas Moleques do Sul), colônias menores de trinta-réis, provavelmente seriam mais eficientes que as grandes. Conseqüentemente, nas ilhas com pequeno contingente desses predadores, pequenas ou grandes colônias podem obter bons desempenhos reprodutivos. Nas proximidades dessas regiões, a atividade pesqueira tem gerado uma valiosa fonte de alimento para aves marinhas, através dos descartes da ictiofauna

Revista Brasileira de Zoologia 20 (4): 655-659, dezembro 2003 
acompanhante do camarão sete-barbas. Esse recurso, em certas épocas do ano, pode representar o principal componente na dieta das aves marinhas (BRANCO 2001).

A análise dos dados de biometria e volume dos ovos sugerem a existência de diferentes populações reprodutoras de $S$. hirundinacea no litoral de Santa Catarina. Essas diferenças, podem estar relacionadas a fatores genéticos das populações, além da disponibilidade de alimento nas proximidades das colônias de reprodução (BRANCO 2001).

\section{AGRADECIMENTOS}

À Universidade do Vale do Itajaí, através do Centro de Ciências Tecnológicas, da Terra e do Mar, pelas facilidades colocadas à disposição durante o andamento deste trabalho. Aos orientandos e estagiários: Hélio Augusto Alves Fracasso, Jan Raphael Reuter Braun, Heder Cassiano Moritz Jr., Bruno Ribeiro Campos, Marcos Siqueira Bovendorp, Irece Farina Machado e Gislei Cibele Bail, entre outros, pelo valioso auxílio nos trabalhos de campo.

\section{REFERÊNCIAS BIBLIOGRÁFICAS}

ANTAS, P.T.Z. 1991. Status and conservation of seabirds breeding in Brazilian waters, p. 141-158. In: J.P. Croxall (Ed.). Seabirds status and conservation: a supplement. Cambridge, ICBP Technical Publication \# 1, 314p.

Bege, L.A.R. \& B.T. Pauli. 1988. As aves nas Ilhas Moleques do Sul - Santa Catarina: Aspectos da ecologia, etologia e anilhamento de aves marinhas. Florianópolis, FATMA, $64 \mathrm{p}$.

BuCKLEY, F.G. \& P.A. BuCKLEY. 1972. The breeding ecology of royal tern Sterna (Thalasseus) maxima maxima. Ibis, London, 114: 344-359.

Burger, J. \& M. GochFeld. 1994. Predation and effects of humans on island-nesting seabirds, p. 39-67. In: D.N. NetTleship; J. Burger \& M. Gochfeld (Eds). Seabirds on Islands. Threats, case studies and action plans. Cambridge, Birdlife International, 318p.

Branco, J.O. 2001. Descartes da pesca do camarão sete-barbas como fonte de alimento para aves marinhas. Revista Brasileira de Zoologia, Curitiba, 18(1): 293-300.

Brown, C.R. \& M.B. Brown. 1987. Group-living in cliff swallows as an advantage in avoiding predators. Behavior Ecology and Sociobiology 21: 97-107.
BRUnTON, D. 1999. "Optimal" colony size for least terns: an intercolony study of opposing selective pressures by predators. The Condor 101: 607-615.

. 1997. Impacts of predators: center nest are less successful than edge nests in a large nesting colony of least terns. The Condor 99: 372-380.

Couson, J.C. 1968. Differences in the quality of birds nesting in the center and on edges of a colony. Nature 217: 478479.

EFe, M.A.; J.L.X. Nascimento; I.L.S. Nascimento \& C. Musso. 2000. Distribuição e ecologia reprodutiva de Sterna sandvicensis eurygnatha no Brasil. Melopsittacus, Belo Horizonte, 3 (3): 110-121.

Furness, R.W. \& P. Monghan. 1987. Seabird ecology. New York, Blackwell, 164p.

Hoyт, D.F. 1979. Practical methods of estimating volume and fresh weight of bird eggs. Auk, Fayetteville, 96: 73-77.

Hunt, G.L.; J.R.Z.A. Eppley \& D.C. Schneider. 1986. Reproductive performance of seabirds: the importance of population and colony size. Auk, Fayetteville, 103: 306-317.

Quintana, F. \& P. Yorio. 1997. Breeding biology of royal and cayenne terns at a mixed-species colony in Patagonia. Wilson Bulletin, Fort Collins, 109 (4): 650-662.

SICK, H. 1997. Ornitologia Brasileira. Rio de Janeiro, Editora Nova Fronteira, 912p.

SICK, H. \& A.P. LẼ̃o. 1965. Breeding sites of Sterna eurygnatha and other sea birds of the Brazilian coast. Auk, Fayetteville, 82: 507-508.

Scolaro, J.A.; S. Laurenti. \& H. Gallelli. 1996. The nesting and breeding biology of the South American Tern in northern Patagonia. Journal of Field Ornithology, Tulsa, 67 (1): 1724.

SOKAL, R.R. \& F.J. Rohlf. 1969. Biometry, the principles and practis of statistics in biological research. San Francisco, W.H. Freeman, 776p.

Yorio, P. \& F. Quintana. 1997. Predation by kelp gulls Larus dominicanus at a mixed-species colony of royal terns Sterna maxima and cayenne terns Sterna eurygnatha in Patagonia. Ibis, London, 139: 536-541.

Yorio, P.; F. Quintana; C. Campagna \& G. Harris. 1994. Diversidad, abundancia y dinamica espacio-temporal de la colonia mixta de aves marinas en Punta Leon, Patagonia. Ornitologia Neotropical, Athens, 6 (2): 69-77.

Recebido em 11.VI.2003; aceito em 28.X.2003. 